\title{
Equação de chuvas intensas para Abaetetuba-PA: estudo comparativo
}

\author{
Intense rainfall equation for Abaetetuba-PA, Brazil: comparative study \\ Curva de lluvia intensa para Abaetetuba-PA, Brasil: estudio comparativo
}

Recebido: 23/03/2021 | Revisado: 29/03/2021 | Aceito: 01/04/2021 | Publicado: 12/04/2021

\author{
Éverton Costa Dias \\ ORCID: https://orcid.org/0000-0003-3842-4688 \\ Universidade Federal do Pará, Brasil \\ E-mail: eng_evertondias@hotmail.com \\ Giovanni Chaves Penner \\ ORCID: https://orcid.org/0000-0003-0335-5352 \\ Universidade Federal do Pará, Brasil \\ E-mail: penner@ufpa.br
}

\begin{abstract}
Resumo
Eventos extremos de chuvas intensas estão se tornando cada vez mais significativos e frequentes no cenário de mudanças climáticas. Este trabalho determinou uma nova equação IDF para o município de Abaetetuba (PA) a partir da série histórica pluviométrica (1980 a 2019) obtida no banco de dados na Agência Nacional de Águas. Os dados avaliados pelos testes de Filliben, de Variância e de Kolmogorov-Smirnov apontaram distribuição Normal com melhor aderência considerado os períodos de retorno 2, 5, 10, 50,100, 200 e 1000 anos. Para a desagregação da precipitação em durações menores que 1 dia se utilizou coeficientes de desagregação recomendados pela literatura. Usando dois métodos de regressão não linear de forma comparativa foram obtidos coeficientes da equação: $K(1197,6964), a(0,0933), b(10,9667)$ e $c(0,7599)$. O confronto da presente equação com a descrita em trabalho anterior aponta intensidades superestimadas pela equação antiga. Desta forma, a equação de chuvas intensas atualizada para Abaetetuba contribuirá no dimensionamento mais econômico de estudos hidráulico-hidrológicos onde seja necessária a determinação da intensidade de precipitação.
\end{abstract}

Palavras-chave: Chuvas intensas; Desagregação; Equação IDF; Regressão não linear.

\begin{abstract}
Heavy rainfall extreme events are becoming increasingly significant and frequent in the climate change scenario. This work determined a new IDF equation for the Abaetetuba municipality (PA) from the historical rainfall series (1980 to 2019) obtained in the database of the National Water Agency. The data evaluated by the Filliben, Variance and Kolmogorov-Smirnov tests showed a Normal distribution with better fit considering the return periods 2, 5, 10, 50, 100, 200 and 1000 years. For the disaggregation of precipitation in lengths shorter than 1 day we used disaggregation coefficients recommended by the literature. Using two methods of non-linear regression in a comparative way were obtained coefficients of the equation: K (1197.6964), a (0.0933), b (10.9667) and c (0.7599). Comparing the present equation with the one described in previous work points to intensities overestimated by the old equation. Thus, the equation of intense rainfall updated for Abaetetuba will contribute to the more economical dimensioning of hydraulichydrological studies where it is necessary to determine the precipitation intensity.
\end{abstract}

Keywords: Intense rainfall; Disaggregation; IDF equation; Nonlinear regression.

\section{Resumen}

Los eventos extremos de lluvia intensa son cada vez más significativos y frecuentes en el escenario de cambios climáticos. En esto trabajo se determinó nueva curva IDF para la municipalidad de Abaetetuba (PA) a partir de la serie histórica de precipitaciones (1980 a 2019) obtenida de la base de datos de la Agencia Nacional del Agua. Los datos evaluados por las pruebas de Filliben, varianza y Kolmogorov-Smirnov indicaron distribución Normal con mejor adherencia considerando los períodos de retorno 2, 5, 10, 50, 100, 200 y 1000 años. Para la desagregación de la precipitación en duraciones menores a 1 día se utilizaron los coeficientes de desagregación recomendados por la literatura. Utilizando dos métodos de regresión no lineal de forma comparativa, se obtuvieron los coeficientes de la ecuación: K $(1197,6964)$, a $(0,0933)$, b $(10,9667)$ y c $(0,7599)$. La comparación de la curva actual con la descrita en un estudio anterior apunta a intensidades sobreestimadas por la curva anterior. Así, la curva de lluvias intensas actualizada para Abaetetuba contribuirá al dimensionamiento más económico de los estudios hidráulico-hidrológicos donde sea necesario determinar la intensidad de las precipitaciones.

Palabras clave: Lluvias intensas; Desagregación; Curva IDF; Regresión no lineal. 


\section{Introdução}

A precipitação é a principal fonte de entrada de água em uma bacia hidrográfica, tornando-se a causa mais importante dos processos hidrológicos que estão dentro da área de interesse da engenharia, caracterizada por uma grande aleatoriedade no tempo e espaço. Da mesma forma, as precipitações intensas ou chuvas extremas, são fenômenos aleatórios e de ocorrência natural, caracterizados por um grande volume precipitado em curtos intervalos de tempo e de forma contínua, cujo intensidades ultrapassam um determinado valor mínimo (Pereira, Duarte \& Sarmento, 2017; Sousa \& Paula, 2018).

Por ser um dos eventos climáticos extremos mais comuns, as chuvas intensas podem desencadear diversos riscos associados, como inundações urbanas, deslizamentos de terra e arraste de sedimentos (Gao, Huang, Chen, Chen, \& Liu, 2017). Segundo Cidades e Soluções (2020), até a exibição do programa “O novo padrão das chuvas intensas no Brasil” em 2020, em um intervalo de 24 horas, as precipitações mais intensas para algumas cidades da região sudeste do Brasil foram: São Paulo (114 $\mathrm{mm}$ ), Belo Horizonte (172 mm, com 60 mortes no Estado de Minas Gerais), Rio de Janeiro (108 mm) e Iconha-ES (210 mm, com 9 mortes no Estado do Espírito Santo). Além disso, situações de inundações urbanas também podem ocorrer quando o sistema de drenagem possui falhas em seu projeto, os quais devem ser dimensionados considerando um evento extremo de precipitação pluviométrica (Rocha Neto \& Blanco, 2020). No meio urbano, as inundações podem ser responsáveis por ocasionar danos socioeconômicos, prejuízos ambientais e até mesmo perdas humanas (Qamar et al., 2017). Dessa forma, para mitigar os efeitos deste fenômeno, diminuindo os prejuízos gerados por eles, as obras hidráulicas, como sistemas de drenagem, devem ser projetadas com base nos valores de vazão máxima, sendo essencial a verificação apropriada das características hidrológicas do local de interesse para a garantia de requisitos mínimos de segurança, durabilidade e eficiência do sistema (Fadhel, Rico-Ramirez, \& Han, 2017; Santos, Fernandes, \& Medeiros, 2019).

O conhecimento de tais vazões, observadas através de análises de dados de precipitação de determinadas durações e frequências, são de fundamental importância em estudos relacionados ao dimensionamentos de projetos hidráulicos (vertedores, canais, barragens e sistemas de drenagem, canais de terraços, drenagem agrícola, urbana e rodoviária, desvio de cursos d’água e práticas mecânicas de controle de erosão hídrica), drenagem urbana e rural (galerias de águas pluviais, bueiros, reservatórios de detenção, vertedores de proteção contra erosões), entre outros (Silva \& Araújo, 2013; Rocha Neto \& Blanco, 2020).

Nesse sentido, a quantificação dessas chuvas pode ser realizada pelo emprego de equações de chuvas intensas, também denominadas de curva de intensidade, duração e frequência (IDF) (Mamoon, Joergensen, Rahman, \& Qasem, 2014; Faridzad, Yang, Hsu, Sorooshian, \& Xiao, 2018). As equações IDF relacionam os volumes precipitados e as intensidades pluviométricas extremas ao período de retorno (tempo necessário para que um evento seja igualado ou superado) associado (Faridzad et al., 2018; Ferreira Filho, Rodrigues, Silva, Fernandes, \& Crispim, 2020).

Campos, Santos, Silva, Filho eLoura (2014) e Svensson, Clarke e Jones (2007) apontam que a determinação das equações IDF deve ser realizada tomando como base os dados de precipitação obtidos por meio de pluviógrafos. Porém, mesmo que estes aparelhos sejam os mais adequados para esse tipo de estudo, a sua utilização é um tanto complexa, visto que as séries registradas, muitas vezes, são curtas e apresentam falhas nos registros de dados (Back, Oliveira, \& Henn, 2012; Dias, Penner, Silva, \& Ribeiro, 2020).

Foi pensando nestas dificuldades que algumas metodologias, conhecidas como desagregação de chuvas diárias, foram desenvolvidas por diversos autores, buscando obter as precipitações de menor duração, a partir dos dados pluviométricos diários (chuvas de 1 dia) (Back et al., 2012). Batista (2013) também indica que a utilização das metodologias de desagregação de chuvas diárias deve ser adotada na ausência de dados de pluviógrafos. A partir destas metodologias a determinação das equações de chuvas intensas se torna mais confiável e acessível aos municípios desprovidos de estações pluviográficas, os quais contam apenas com estações pluviométricas, como é o caso de Abaetetuba. 
Portanto, devido a importância que os estudos de chuvas intensas têm para a melhoria da qualidade dos projetos hidráulico-hidrológicos nos municípios brasileiros e do mundo, este trabalho buscou determinar uma equação IDF para o município de Abaetetuba (PA) a partir da série histórica pluviométrica atualizada, obtida no banco de dados na Agência Nacional de Águas (ANA).

\section{Metodologia}

\subsection{Caracterização da área de estudo}

De acordo com o Instituto Brasileiro de Geografia e Estatística (IBGE), o município de Abaetetuba-PA localiza-se a 60 km a sudoeste da capital Belém, nas coordenadas geográficas centrais de $01^{\circ} 43^{\prime} 24^{\prime \prime}$ de latitude Sul e $48^{\circ} 52^{\prime} 54^{\prime}$ ” de longitude Oeste. No ano de 2010, Abaetetuba congregava 141.100 habitantes, com uma estimativa de 157.698 habitantes para 2019. Possui extensão territorial de 1.610,652 km² (zona urbana e rural), sendo que a sede do município é composta por 14 bairros (São Lourenço, Francilândia, Centro, Aviação, São Sebastião, Cristo Redentor, São José, São João, São Domingos de Angélica, Santa Rosa, Mutirão, Santa Clara, Castanhal e Algodoal) (Dias, Silva, Almeida \& Fernandes, 2018). Situado na região amazônica, Abaetetuba possui grande abundância de rios bem como ampla vegetação, sendo que na área urbana o índice de arborização em vias públicas alcança 26\% (IBGE, 2020). Por estar próximo à linha do Equador, considera-se uma área tipicamente de clima equatorial, ou seja, quente e úmido. Na Figura 1 indica-se a localização do município em questão.

Figura 1. Mapa de localização do município de Abaetetuba - PA.

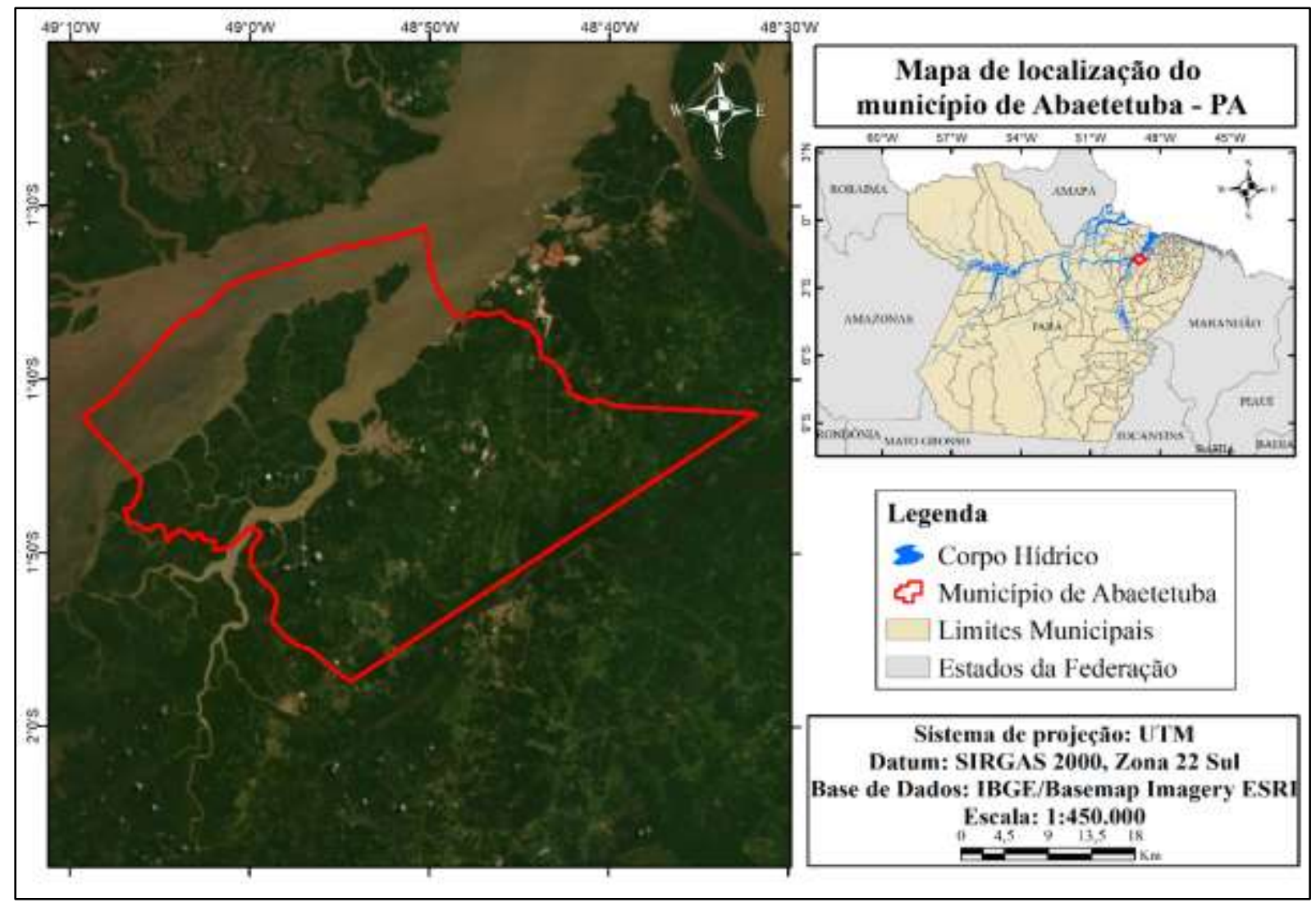

Fonte: Autores (2021).

\subsection{Coleta e análise de dados}

A série histórica de chuvas máximas de 1 dia do município de Abaetetuba utilizada neste estudo foi obtida a partir do banco de dados Hidroweb (Sistema de Informações Hidrológicas) versão v3.1.1, da ANA. A estação pluviométrica é de 
responsabilidade da ANA, porém a sua operação é realizada pela Companhia de Pesquisa de Recursos Minerais (CPRM). Seu código é 148010 e está situada nas coordenadas $1^{\circ} 45^{\prime} 1.14 " \mathrm{~S}$ e 4852'0.83"O (ANA, 2020), conforme Figura 2.

Figura 2. Localização da estação pluviométrica de Abaetetuba.

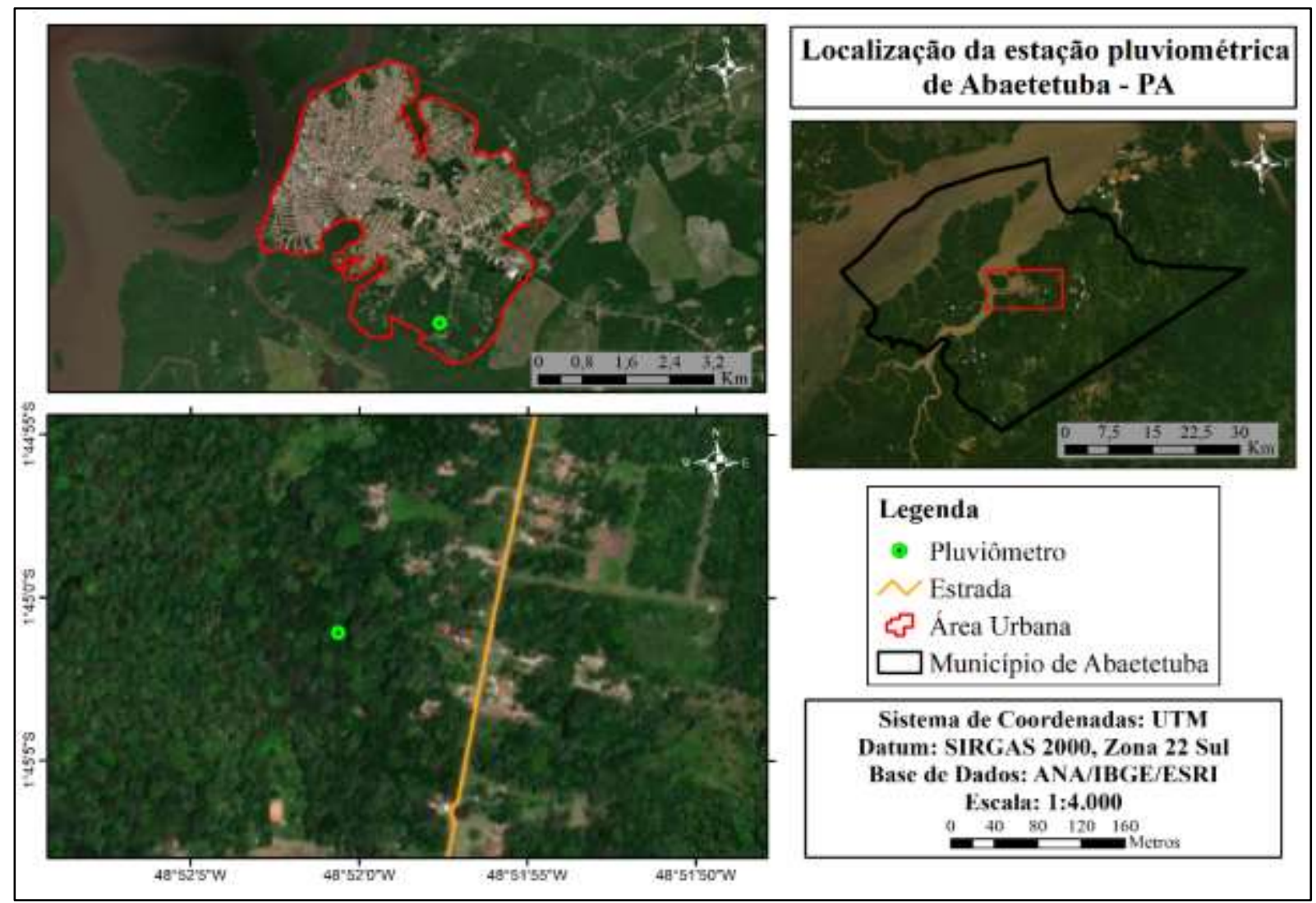

Fonte: Autores (2021).

A série histórica de chuvas diárias obtida conta com 40 anos de dados (1980 - 2019). Porém, dentro deste período há alguns anos que possuem falhas em alguns meses, situação comum em qualquer rede de monitoramento, de forma que foram considerados apenas os maiores valores de precipitação diária observados em cada ano. Para avaliar a necessidade de exclusão de algum ano com falhas, verificou-se se a maior precipitação diária observada neste ano era superior a pelo menos um dos valores de precipitação máxima diária obtido nos anos sem falhas. Todos os anos com falhas foram aprovados por este critério, portanto, todas as precipitações máximas dos 40 anos da série histórica puderam ser utilizadas. Sendo assim, não foi necessária a utilização de nenhum método de preenchimento de falhas para a série histórica analisada.

Como a estação pluviométrica de Abaetetuba possui uma série histórica de 40 anos de dados, também atende à recomendação da World Meteorological Organization de dispor de ao menos 30 anos de dados para que se tenha uma confiabilidade na caracterização climática de uma região.

\subsection{Aplicação dos métodos}

Para a análise estatística utilizou-se o programa SEAF (Sistema Especialista em Análise de Frequência) 1.0, desenvolvido pelo Departamentos de Engenharia Sanitária e Ambiental e Engenharia Hidráulica e Recursos Hídricos da Universidade Federal de Minas Gerais.

Neste caso, foi conduzida uma análise de frequência seguindo as seguintes etapas intermediárias: verificação da presença de valores atípicos (outliers); avaliação da independência e da homogeneidade da série; aplicação das distribuições 
teóricas de probabilidades candidatas a modelagem das intensidades de chuva; cálculo dos parâmetros das distribuições teóricas de probabilidades candidatas (Normal, Log-Normal, Gumbel, Exponencial, Generalizada de Valores Extremos (GEV), Generalizada de Pareto, Pearson III e Log-Pearson III); definição da(s) distribuição(ões) teórica(s) a ser adotada na modelagem das séries a partir da verificação da aderência à distribuição empírica; e, estimação dos quantis associados a diferentes períodos de retorno (Pinto, 2013).

\subsubsection{Teste de aderência}

Para que o SEAF indique qual a distribuição de frequência possui melhor aderência dos dados observados aos dados calculados, este utiliza os testes estatísticos de ajuste de Filliben e de Variância a um nível de significância de $10 \%(\alpha=0,1)$.

Adicionalmente usando planilha eletrônica Microsoft Excel 2016 foi aplicado o teste de Kolmogorov-Smirnov (K-S). Para realização dos testes foi considerado um nível de significância de $5 \%(\alpha=0,05)$, sendo este valor bastante utilizado em estudos para determinação de equações de chuvas intensas, tais como os de Miranda, Thebaldi, \& Rocha (2017); Basumatary \& Sil (2017); Shrestha, Babel, Weesakul, e Vojinovic (2017) e Sane et al. (2018).

Aliado ao teste de K-S, também foram utilizadas as seguintes posições de plotagem: Weibull, Mediana, Hosking, Blom, Cunnane, Gringorten e Hazen; para determinar o máximo módulo da diferença das probabilidades acumuladas e conferir se a hipótese da distribuição teórica representa a distribuição empírica ao nível de significância 5\%, conforme é indicado em Naghettini e Pinto (2007).

\subsection{Desagregação da chuva diária}

Para a desagregação das chuvas diárias, observada em pluviômetro, em alturas pluviométricas de menor duração lançouse mão dos coeficientes de desagregação recomendados por Occhipinti e Santos (1966), sugeridos em CETESB (1986), ver Tabela 1, e amplamente utilizados no Brasil: Back (2009); Back et al. (2012); Cardoso, Ullmann, e Bertol (1998); Genovez e Zuffo (2000); e Silveira (2000). Ressalta-se que em seu estudo, Martins, Gandini, Kruk, e Queiroz (2019), obtiveram coeficientes diferentes destes.

Com a desagregação foram obtidas as alturas de precipitações para durações menores: 5, 15, 30, 60, 120, 360, 720, e 1440 minutos, fornecendo dados para a determinação das curvas IDF, considerando os tempos de retorno de 2, 5, 10, 50, 100, 200 e 1000 anos.

Tabela 1. Relação entre durações e coeficientes de desagregação.

\begin{tabular}{c|c}
\hline Relação entre durações & Fator de desagregação \\
\hline $5 \mathrm{~min} / 30 \mathrm{~min}$ & 0,34 \\
$15 \mathrm{~min} / 30 \mathrm{~min}$ & 0,70 \\
$30 \mathrm{~min} / 1 \mathrm{~h}$ & 0,74 \\
$1 \mathrm{~h} / 24 \mathrm{~h}$ & 0,42 \\
$2 \mathrm{~h} / 24 \mathrm{~h}$ & 0,48 \\
$6 \mathrm{~h} / 24 \mathrm{~h}$ & 0,72 \\
$12 \mathrm{~h} / 24 \mathrm{~h}$ & 0,85 \\
$24 \mathrm{~h} / 1 \mathrm{dia}$ & 1,14 \\
\hline
\end{tabular}

Fonte: modificado de CETESB (1986).

\subsection{Determinação da Equação IDF de Abaetetuba}

Sabe-se que a equação mais utilizada para expressar a relação intensidade, duração e frequência é representada conforme Equação 1: 
$\mathrm{i}=\frac{\mathrm{K} \cdot \mathrm{T}_{\mathrm{r}}^{\mathrm{a}}}{(\mathrm{t}+\mathrm{b})^{\mathrm{c}}}$

Em que:

i é a intensidade da precipitação $(\mathrm{mm} / \mathrm{h})$;

Tr é o tempo de retorno (anos);

t é a duração da precipitação (minutos);

K, a, b, c são os coeficientes da equação que descrevem características locais (adimensional).

Com a forma da equação IDF definida, deu-se prosseguimento ao processamento dos dados de precipitação, estimando os coeficientes da equação para o município de Abaetetuba. Para tanto, utilizou-se a metodologia de regressão não-linear, também conhecida como método Gauss - Newton, minimizando a Soma dos Quadrados dos Resíduos (SQRes), por planilha eletrônica do Microsoft Excel 2016, conforme recomendado em Penner e Lima (2016).

Comparativamente aplicou-se regressão não-linear seguindo a metodologia de Pereira, Duarte e Sarmento (2017), sendo usada a ferramenta computacional Solver do programa Microsoft Excel 2016 para determinar os parâmetros $(K, a, b$ e $c)$ da Equação 1, também buscando minimizar a SQRes. Vale destacar que tal metodologia também foi aplicada por Sousa \& Paula (2018).

\subsection{Teste de validação dos parâmetros}

Como forma de validar o quão próximos estão os valores de intensidade de precipitação calculadas dos valores de intensidade observados, empregou-se quatro diferentes parâmetros, sendo eles: o Índice de Desempenho (C), proposto por Camargo e Sentelhas (1997); o Índice de Willmott (d); o Coeficiente de Correlação (R); e por fim, o Coeficiente de Determinação $\left(\mathrm{R}^{2}\right)$, sendo os dois últimos amplamente conhecidos e bastante utilizado no meio estatístico.

O índice de concordância de Wilmott (d) determina a exatidão do método utilizado, e avalia o grau de afastamento entre os valores estimados e observados, variando também entre 0 e 1 . Como critério de interpretação do Índice de Desempenho (Ic) obtido foi utilizada a Tabela 2, proposta por Camargo e Sentelhas (1997).

Tabela 2. Critérios de avaliação do Índice de desempenho C.

\begin{tabular}{c|c}
\hline Índice C & Desempenho \\
\hline$>0,85$ & Ótimo \\
0,76 a 0,85 & Muito bom \\
0,66 a 0,75 & Bom \\
0,61 a 0,65 & Mediano \\
0,51 a 0,60 & Sofrível \\
0,41 a 0,50 & Mau \\
$\leq 0,40$ & Péssimo \\
\hline
\end{tabular}

Fonte: Adaptado de Camargo e Sentelhas (1997).

\section{Resultados e Discussões}

\subsection{Caracterização da precipitação em Abaetetuba}

Verificou-se, inicialmente, que as máximas precipitações pluviométricas diárias foram registradas nos dias: 3 de fevereiro de 1995 (180,8 mm), 26 de janeiro de 1988 (144,8 mm) e 29 de dezembro de 1999 (131 mm). Por outro lado, os menores valores foram observados nos dias: 20 de fevereiro de 1983 (44,2 mm), 31 de dezembro de 1980 (45 mm) e 12 de abril de 1985 (47,2 mm). A série apresentou média de chuva máxima diária anual de 93,25 mm e desvio padrão de 29,46 mm. 


\subsection{Seleção da distribuição probabilística}

O teste de Kendall e Mann-Kendall, realizados no software SEAF 1.0, apontaram que os dados são dependentes e não homogêneos, respectivamente. A dependência significa a existência de correlação entre o registro de um dado ano e os registros posterior e anterior, considerados todos os anos disponíveis. Já a não homogeneidade pode estar associada mudanças abruptas na tendência dos dados.

Não foram identificados outliers máximos e mínimos pelo teste de Grubbs e Beck. Entretanto, o teste de assimetria para o limite superior aponta que há outlier, isto é, a retirada do máximo amostral resultou em uma mudança significativa da assimetria. Pelo exposto conferiu-se que o valor máximo observado, 180,8 mm, havia sido consistido em segundo nível. Consultando a estação pluviométrica de Barcarena, cidade vizinha, verificou-se que também há um registro de precipitação intensa no dia anterior ao observado em Abaetetuba, sendo que em 2 de fevereiro de 1995 a precipitação foi 96,8 mm, registrada como máxima do referido ano. Portanto, não se descartou o registro.

Para verificar a melhor aderência dentre as distribuições probabilísticas aos dados empíricos foram aplicados os testes de Filliben e de Variância sendo os resultados indicados na Tabela 3.

Tabela 3. Seleção da melhor distribuição probabilística.

\begin{tabular}{lccc}
\hline \multicolumn{1}{c}{ Distribuições Probabilísticas } & \multicolumn{2}{c}{ Teste de Filliben } & \multirow{2}{*}{ Teste da Variância $|Z|$} \\
\hline Normal & R $(90 \%)$ & $\mathrm{R}$ & 0,4311 \\
LogNormal & 0,9765 & 0,9795 & 1,7965 \\
Gumbel & 0,9765 & 0,9717 & 1,7055 \\
Exponencial & 0,9692 & 0,9776 & 3,6817 \\
Pearson-III & 0,9617 & 0,9364 & 0,4614 \\
LogPearson-III & 0,9835 & 0,9822 & 0,2802 \\
GEV & 0,9804 & 0,9773 & 0,7139 \\
GPA & 0,9848 & 0,9809 & 3,2827 \\
\hline
\end{tabular}

Fonte: Autores (2021).

Comparando o coeficiente de correlação do teste de Filliben (R) e seu respectivo intervalo unilateral a 90\% de confiança R (90\%), para uma distribuição ser aceita precisa satisfazer a condição R > R (90\%). Tal comparação resulta na aceitação das distribuições Normal e Gumbel, rejeitando as demais.

Já o teste da Variância, que determina o coeficiente de variação, $|\mathrm{Z}|$, indicando quanto mais próximo a zero melhor é o ajuste da distribuição aos dados empíricos. Neste caso, comparando-se as distribuições selecionadas pelo teste de Filliben, a distribuição Normal é a melhor.

Para finalizar a seleção da melhor distribuição probabilista, foi aplicado o teste K-S para as distribuições Normal e Gumbel, testando as posições de plotagem mencionadas. Neste teste foi determinado o máximo módulo da diferença das probabilidades acumuladas (D), o qual precisa ser inferior a diferença fornecida pelo teste K-S para distribuição não ser rejeitada $\left(D_{N, \alpha \%}\right)$. Neste caso, para um tamanho amostral igual a $40(\mathrm{~N}=40)$, e para um nível de significância de $5 \%(\alpha=0,05)$, o teste $\mathrm{K}$ $\mathrm{S}$ forneceu o valor de $\mathrm{D}_{40 ; 5 \%}=0,215$. A distribuição Normal com a posição de plotagem Cunnane apresentou $\mathrm{D}=0,07317 \mathrm{e}$ a distribuição Gumbel com a posição de plotagem de Hazen apresentou D =0,13236. Desta forma, ambas as distribuições são

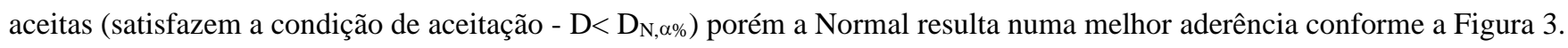


Figura 3. Teste K-S para as distribuições Gumbel (3a) e Normal (3b).

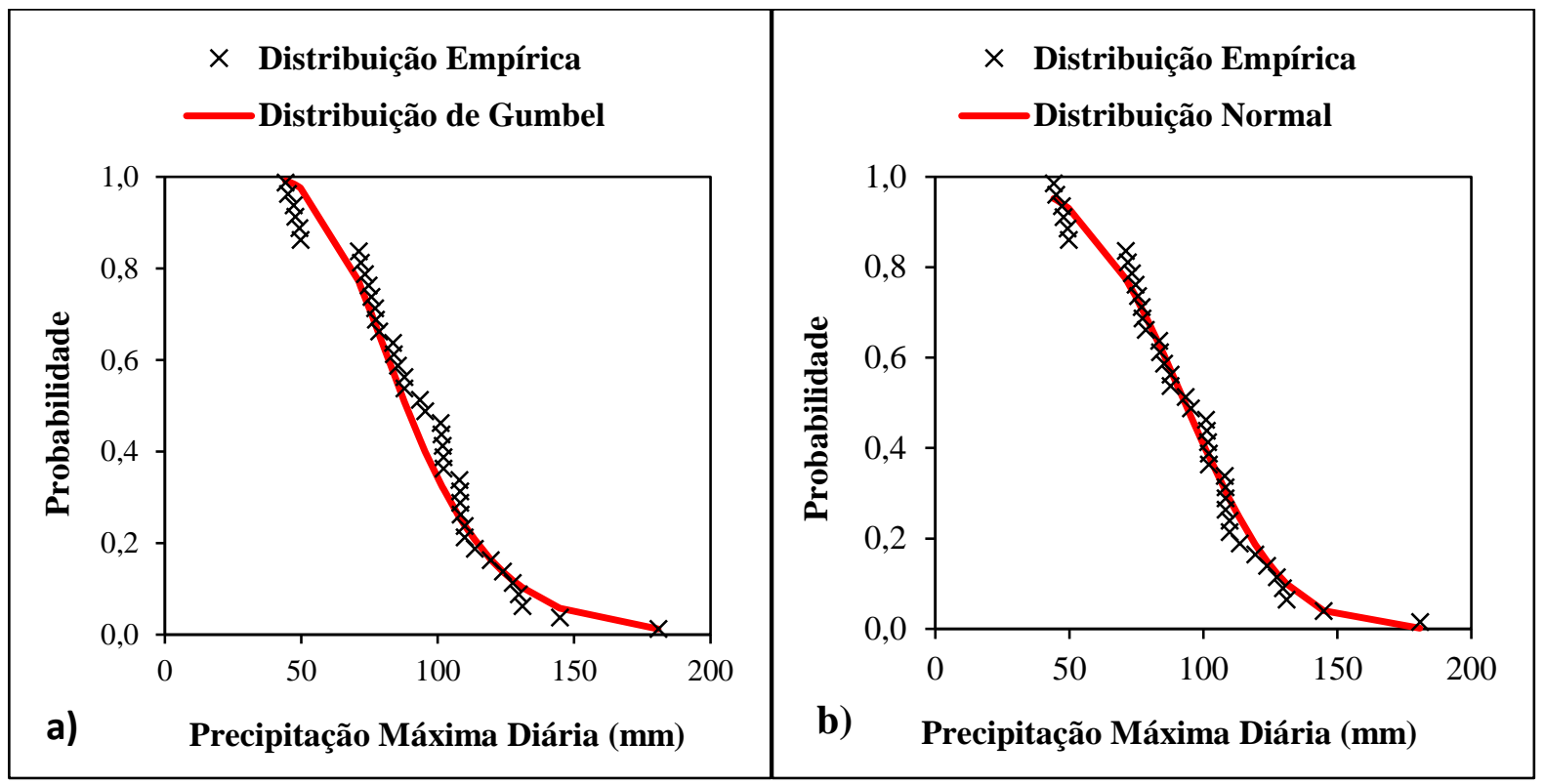

Fonte: Autores (2021).

\subsection{Determinação dos parâmetros da Equação IDF}

Como a distribuição Normal apresentou a melhor aderência, utilizou-se as precipitações de um dia para os diferentes períodos de retorno obtidos a partir do software SEAF 1.0 para esta distribuição. As precipitações de um dia foram desagregadas para as durações menores seguindo CETESB (1986). Sendo os coeficientes $K, a, b$ e $c$ da equação de chuvas intensas obtidos usando duas metodologias distintas: uma proposta em Penner r Lima (2016) e a outra proposta por Pereira et al. (2017). Na Tabela 4 está arrolado o resumo da aplicação de ambas as metodologias para a determinação dos coeficientes da equação IDF, assim como os índices e coeficientes de validação necessários para que seja analisada a qualidade do ajuste entre as intensidades; sendo Penner r Lima (2016) identificada como Normal (1) e Pereira et al. (2017) identificada como Normal (2).

Tabela 4. Coeficientes da equação IDF e parâmetros de validação obtidos por diferentes metodologias.

\begin{tabular}{lccc}
\hline Indicador & Símbolo & Normal (1) & Normal (2) \\
\hline Coeficiente IDF & $\mathrm{K}$ & 1199,4393 & 1197,6964 \\
Coeficiente IDF & $\mathrm{a}$ & 0,0933 & 0,0933 \\
Coeficiente IDF & $\mathrm{b}$ & 10,9791 & 10,9667 \\
Coeficiente IDF & $\mathrm{c}$ & 0,7602 & 0,7599 \\
Coeficiente de Determinação & $\mathrm{R}^{2}$ & 0,9937 & 0,9937 \\
Coeficiente de Correlação & $\mathrm{R}$ & 0,9968 & 0,9968 \\
Índice de Concordância de Willmott & $\mathrm{d}$ & 0,9984 & 0,9984 \\
Índice de Confiança & $\mathrm{Ic}$ & 0,9952 & 0,9952 \\
\hline
\end{tabular}

Fonte: Autores (2021).

Analisando os resultados da Tabela 4 fica evidente que as metodologias para determinação dos coeficientes da equação IDF fornecem resultados muito próximos ou idênticos. Além disso, os coeficientes de Determinação e Correlação, e índices de Concordância e Confiança, usados para validação da equação IDF por ambas as metodologias, foram quase idênticos, sendo observada variação na ordem $10^{-6}$, de forma que a metodologia proposta por Pereira et al. (2017) resultou em melhor ajuste. Os coeficientes " $R$ " e " $R$ " são ligeiramente inferiores aos de Sousa e Paula (2018), assim como os índices "d" "Ic" e " $R$ "” também 
foram inferiores aos de Pereira et al. (2017). Todavia, o resultado obtido para o Coeficiente de Determinação neste estudo foi melhor que o de Teodoro, Neivock, Marques, Flores e Braga (2014).

Pelo exposto, substituindo os coeficientes da coluna Normal 2 (Tabela 4) na Equação 1 tem-se a equação atualizada de intensidade, duração e frequência (IDF) para o município de Abaetetuba (Equação 2):

$i=\frac{1197,6964 \cdot \operatorname{Tr}^{0,0933}}{(t+10,9667)^{0,7599}}$

A partir das intensidades estimadas pode-se gerar as curvas IDF para o município de Abaetetuba (Figura 4). Nesta figura, quando se busca analisar as variáveis intensidade e duração de precipitação, pode-se, então, verificar que existe uma relação inversamente proporcional entre ambas, para um mesmo tempo de retorno. Dessa forma, quanto maior for a duração, menor será a intensidade de chuva obtida. Por outro lado, para uma mesma duração, a relação passa a ser diretamente proporcional entre o tempo de retorno e a intensidade, ou seja, maior será a intensidade de precipitação quanto maior for o período de retorno. Portanto, analisando as três variáveis separadamente, maior será a intensidade de precipitação quanto menor for a duração e maior for o tempo de retorno. Relações semelhantes a estas também são verificadas em Dias et al. (2020).

Figura 4. Curvas IDF para o município de Abaetetuba-PA.

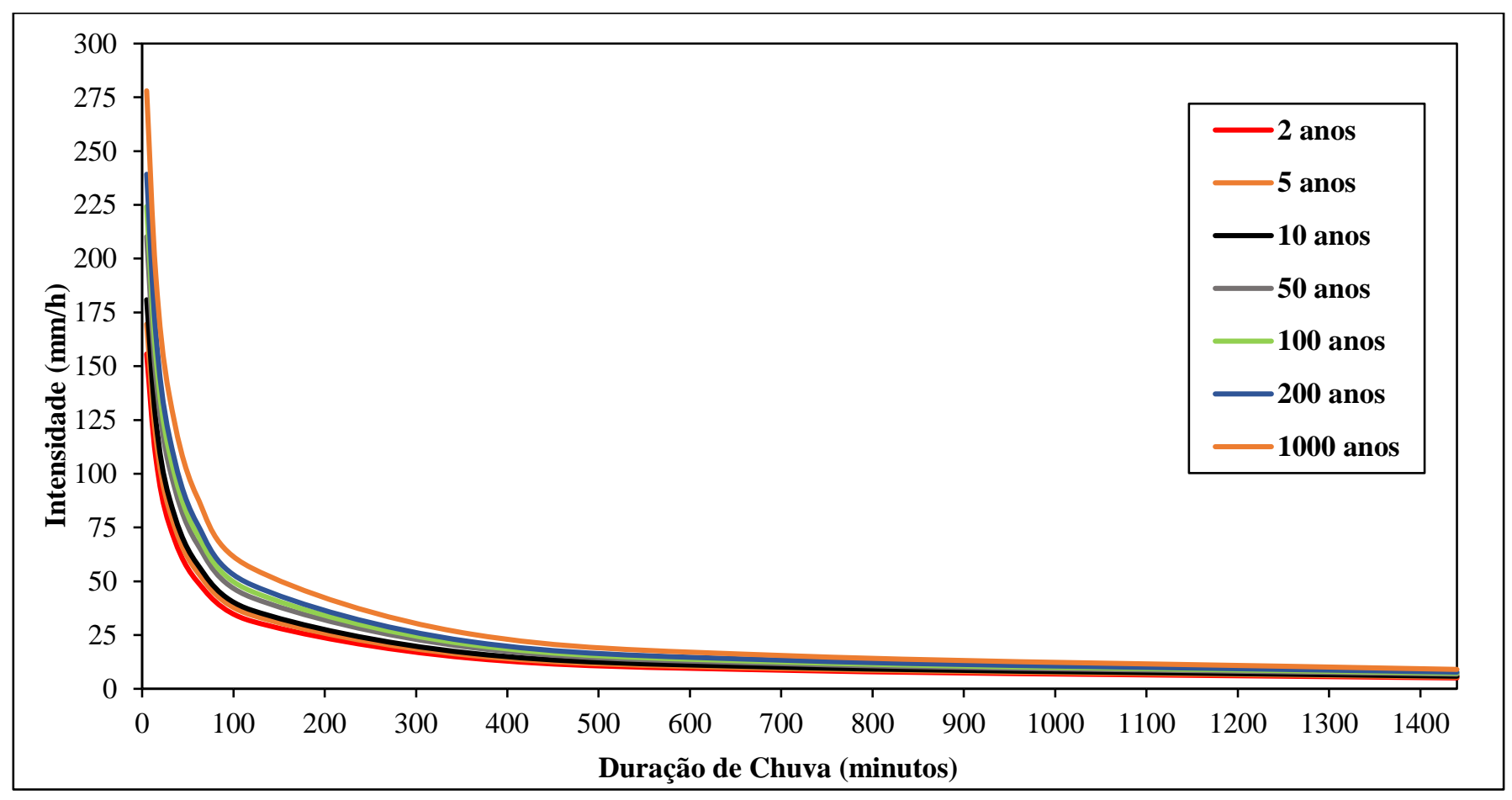

Fonte: Autores (2021).

\subsection{Avaliação da equação IDF proposta}

Como forma de avaliar os coeficientes propostos na Equação 2, apresenta-se, a partir da Figura 5, a relação entre as intensidades observadas e estimadas, bem como a linha de tendência e o coeficiente $\mathrm{R}^{2}$, confirmando o reduzido desvio entre ambas. 
Figura 5. Relação entre intensidade de precipitação observada e calculada, linha de tendência e o valor de $\mathrm{R}^{2}$.

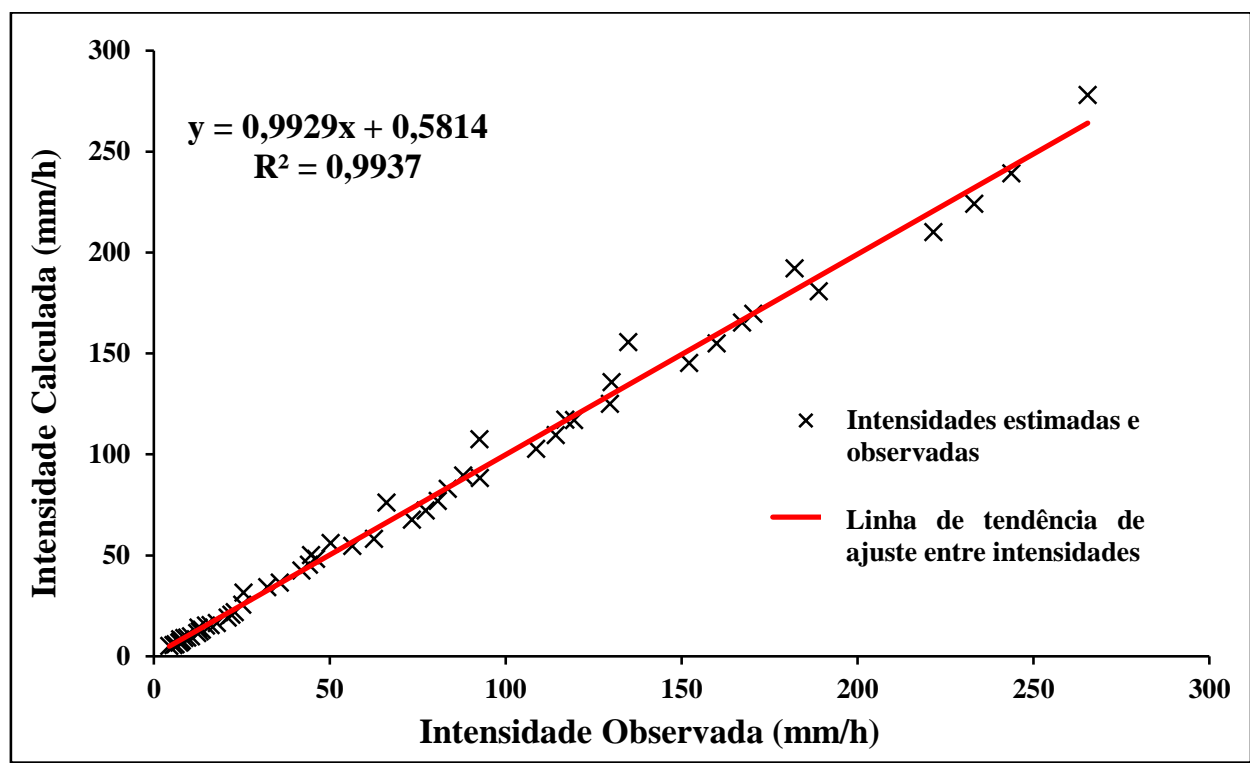

Fonte: Autores (2021).

A partir dos valores de intensidades observadas e estimadas gerou-se a Tabela 5, a qual apresenta os erros percentuais encontrados entre as duas intensidades.

Tabela 5. Erro percentual entre intensidades estimadas e observadas.

\begin{tabular}{c|ccccccc}
\hline \multirow{2}{*}{ Duração (min) } & \multicolumn{7}{c}{$\operatorname{Tr}$ (anos) } \\
& 2 & 5 & 10 & 50 & 100 & 200 & 1000 \\
\hline 5 & 15,48 & $-0,48$ & $-4,28$ & $-5,15$ & $-3,81$ & $-1,82$ & 4,74 \\
15 & 16,28 & 0,21 & $-3,61$ & $-4,49$ & $-3,14$ & $-1,14$ & 5,47 \\
30 & 15,13 & $-0,78$ & $-4,57$ & $-5,44$ & $-4,10$ & $-2,12$ & 4,43 \\
60 & 12,23 & $-3,28$ & $-6,97$ & $-7,81$ & $-6,51$ & $-4,58$ & 1,80 \\
120 & 23,30 & 6,26 & 2,20 & 1,27 & 2,70 & 4,83 & 11,84 \\
360 & 11,79 & $-3,66$ & $-7,34$ & $-8,18$ & $-6,89$ & $-4,96$ & 1,40 \\
720 & 13,11 & $-2,52$ & $-6,24$ & $-7,09$ & $-5,78$ & $-3,83$ & 2,60 \\
1440 & 14,21 & $-1,58$ & $-5,33$ & $-6,19$ & $-4,87$ & $-2,90$ & 3,59 \\
\hline Média & 15,19 & $-0,73$ & $-4,52$ & $-5,38$ & $-4,05$ & $-2,07$ & 4,48 \\
\hline
\end{tabular}

Fonte: Autores (2021).

A partir da Tabela 5 verifica-se que a Equação 2 apresenta tendência a superestimar as intensidades quando o período de retorno atinge seu limite inferior e superior ( 2 e 1000 anos), fato que é indicado pelos erros percentuais positivos. Nos outros casos, de uma forma geral, as intensidades são ligeiramente subestimadas. Vale ressaltar que, considerando todos os resultados, o erro médio é de 0,42\%, indicando que as intensidades estimadas são, em geral, 0,42\% maiores que as observadas. Desta forma, tem-se um equilíbrio entre valores subestimados e superestimados. Adicionalmente os maiores erros foram direcionados ao período de retorno 2 anos e as durações 15 min e 120 min $(2$ h). Destaca-se que tais erros são superiores aos observados por Pereira et al. (2017).

Quando se compara as intensidades calculadas para Abaetetuba por Souza et al. (2012), utilizando seus respectivos coeficientes $(\mathrm{K}=1086,3999 ; \mathrm{a}=0,1193 ; \mathrm{b}=9,7855 ; \mathrm{c}=0,7242)$, com as atuais indicadas neste estudo, verifica-se que todas as intensidades obtidas por estes autores são superestimadas, com média geral de $17,5 \%$. Sendo assim, tem-se que a equação proposta subestima as intensidades de chuva para Abaetetuba. Porém, vale ressaltar que tal fato não a torna inválida, pelo contrário, o seu desenvolvimento demonstra a necessidade constante de atualização das equações de chuvas intensas, de forma 
a contribuir com um dimensionamento hidrológico mais adequado das estruturas de drenagem, amenizando problemas como alagamentos.

\section{Conclusões}

Em trabalhos que objetivem a elaboração da equação de chuvas intensas é fundamental a determinação da distribuição probabilística que possua melhor aderência aos dados obtidos. Tal constatação ficou evidente no presente estudo, no qual a distribuição Normal foi eleita como mais adequada para ser utilizada, diferentemente do que é encontrado em literatura especializada, já que é frequente o uso da distribuição Gumbel para as máximas chuvas diárias. Porém, confirmou-se que a distribuição Gumbel nem sempre é a eleita, mostrando a importância do teste da melhor distribuição para que se tenha a melhor predição.

A metodologia aplicada neste estudo, para a determinação das relações de intensidade, duração e frequência das chuvas intensas, mostrou-se acessível e de confiável aplicabilidade para o município de Abaetetuba-PA, o qual conta somente com registro de pluviômetro. Por outro lado, é necessário que a rede pluviográfica seja cada vez mais ampliada e seus dados disponibilizados, principalmente na região Norte, a qual tem uma cobertura reduzida. Neste caso, evitar-se-ia a necessidade do uso de coeficientes de desagregação, os quais, provavelmente, não representam de forma fiel a realidade para todas as localidades do país, como já discutido pela literatura especializada.

Após a aplicação da ferramenta computacional Solver, Microsoft Excel 2016, para determinação dos parâmetros da equação IDF, verificou-se que os valores obtidos para os diferentes coeficientes indicadores da aderência da equação aos dados observados $\left(\mathrm{R}^{2}, \mathrm{R}, \mathrm{d}, \mathrm{Ic}\right)$ apontaram excelente ajuste, superior a 0,99 , com a convergência dos coeficientes $(K, a, b$ e $c)$ da equação construída associados a um erro de truncamento superior a 10-6. Sendo a metodologia proposta por Pereira et al. (2017) a qual resultou em melhor ajuste e a eleita para aplicação no presente trabalho.

Por fim, a média geral do erro percentual entre intensidades estimadas e observadas foi de $0,42 \%$, indicando ótima qualidade da equação construída na predição dos dados observados, e quando comparadas as intensidades estimadas com aquelas obtidas a partir da equação proposta por Souza et al. (2012) tal valor foi 17,5\% inferior. Tal condição evidencia a importância da constante atualização dos coeficientes da equação IDF, visto que os custos de execução de uma obra que passe pelo dimensionamento hidráulico-hidrológico serão majorados se a precipitação extrema calculadas estiver superestimada. Aliado a esta constatação, os efeitos das mudanças climáticas estão se tornando cada vez mais frequentes, com eventos extremos mais acentuados, sendo mais uma justificativa para a periódica atualização das equações IDF.

\section{Referências}

Agência Nacional de Água - ANA. (2020). Séries Históricas de Estações. http://www.snirh.gov.br/hidroweb/serieshistoricas.

Back, A. J. (2009). Relações entre precipitação intensas de diferentes durações ocorridas no município de Urussanga, SC. Revista Brasileira de Engenharia Agrícola e Ambiental, 13(2), 170-175.

Back, A. J., Oliveira, J. L. R., \& Henn, A. (2012). Relações entre precipitações intensas de diferentes durações para desagregação da chuva diária em Santa Catarina. Revista Brasileira de Engenharia Agrícola e Ambiental, 16 (4), 391-398.

Basumatary, B., \& Sil, B. S. (2017). Generation of Rainfall Intensity-Duration-Frequency curves for the Barak River Basin. Meteorology Hydrology and Water Management, 6(1), 01-11.

Batista, M. L. (2013). Precipitação máxima diária anual na região Sudeste do Brasil: distribuição de probabilidade e análise espacial. (Dissertação de Mestrdo). Programa de Pós-Graduação em Recursos Hídricos em Sistemas Agrícolas, Universidade Federal de Lavras (UFLA), Lavras, MG, Brasil.

Camargo, A. P., \& Sentelhas, P. C. (1997). Avaliação do desempenho de diferentes métodos de estimativa da evapotranspiração potencial no estado de São Paulo, Brasil. Revista Brasileira de Agrometeorologia, 5(1), 89-97.

Campos, A. R., Santos, G. G., Silva, J. B. L., Filho, J. I., \& Loura, D. S. (2014). Equações de Intensidade-Duração-Frequência de chuvas para o Estado do Piauí. Revista Ciência Agronômica, 45(3), 488-498. 
Cardoso, C. O., Ullmann, M. N., \& Bertol, I. (1998). Análise de chuvas intensas a partir da desagregação das chuvas diárias de Lages e de Campos Novos (SC). Revista Brasileira de Ciências do Solo, 22, 131-140.

CETESB. (1986). Departamento de Água e Energia Elétrica-Companhia de Tecnologia de Saneamento Ambiental. Drenagem urbana: Manual de projeto. São Paulo, Brasil: DAEE/ CETESB.

Cidades \& Soluções. (2020). O novo padrão das chuvas intensas no Brasil. http://g1.globo.com/globo-news/clube-dos-correspondentes/videos/t/outrosprogramas/v/o-novo-padrao-das-chuvas-intensas-no-brasil/8330718/.

Dias, E. C., Silva, G. P. C., Almeida, I. R., \& Fernandes, L. L. (2018). Proposta de dimensionamento de um sistema de abastecimento de água para o bairro de Algodoal no município de Abaetetuba-Pará. Periódico Tchê Química, 15(30), 347-356.

Dias, E. C., Penner, G. C., Silva, G. P. C., \& Ribeiro, R. P. (2020). Utilização de dados pluviométricos diários para determinação da equação IDF para o município de Abaetetuba - Pará. Brazilian Journal of Development, 6, 8694-8709.

Fadhel, S., Rico-Ramirez, M. A., \& Han, D. (2017). Uncertainty of Intensity-Duration-Frequency (IDF) curves due to varied climate baseline periods. Journal of Hydrology, 547, 600-612.

Faridzad, M., Yang, T., Hsu, K., Sorooshian, S., \& Xiao, C. (2018). Rainfall Frequency Analysis for Ungauged Regions using Remotely Sensed Precipitation Information. Journal of Hydrology, 563, 123-142.

Ferreira Filho, D. F., Rodrigues, R. S. S., Silva, M. N. A., Fernandes, L. L. \& Crispim, D. L. (2020). Aplicação de diferentes métodos de determinação de curvas de intensidade-duração frequência no município de Belterra no estado do Pará, Brasil. Research, Society and Development, 9, 1-26.

Gao, L., Huang, J., Chen, X., Chen, Y., \& Liu, M. (2017). Risk of extreme precipitation under nonstationarity conditions during the second flood season in the Southeastern Coastal Region of China. Journal of Hydrometeology, 18: 669-681.

Genovez, A. M., \& Zuffo, A. C. (2000). Chuvas Intensas no Estado de São Paulo: Estudos Existentes e Análise Comparativa. Revista Brasileira de Recursos Hídricos, 5(3), 45-58.

Instituto Brasileiro de Geografia e Estatítica (IBGE). Cidades - Abaetetuba. https://cidades.ibge.gov.br/brasil/pa/abaetetuba/panorama.

Mamoon, A. A., Joergensen, N. E., Rahman, A., \& Qasem, H. (2014). Derivation of new design rainfall in Qatar using L-moment based index frequency approach. International Journal of Sustainable Built Environment, 3(1), 111-118.

Martins, D., Gandini, M. L. T., Kruk, N. S., \& Queiroz, P. I. B. (2019). Disaggregation of daily rainfall data for the Caraguatatuba city, in São Paulo State, Brazil. Revista Brasileira de Recursos Hídricos, 24, 1-9.

Miranda, C. T. S., Thebaldi, M. S., \& Rocha, G. M. R. B. (2017). Precipitação máxima diária anual e estimativa da equação de chuvas intensas do município de Divinópolis, MG, Brasil. Scientia Agrária, 18(4), 09-16.

Naghettini, M., \& Pinto, E. J. A. (2007). Hidrologia estatística. CPRM.

Penner, G. C., \& Lima, M. P. (2016). Comparação entre métodos de determinação da equação de chuvas intensas para a cidade de Ribeirão Preto. Geocincias, $35(4), 542-559$

Pereira, D. C., Duarte, L. R., \& Sarmento, A. P. (2017). Determinação da curva de intensidade, duração e frequência do município de Ipameri - Goiás. Revista Eletrônica de Engenharia Civil, 13(2), 233-246.

Pinto, E. J. A. (2013). Atlas Pluviométrico do Brasil - metodologia para definição das equações intensidade-duração-frequência do Projeto Atlas Pluviométrico. Belo Horizonte, MG: CPRM.

Occhipinti, A. G., \& Santos, P. M. (1966). Relação entre as precipitações máximas de "um dia" e de "24 horas" na cidade de São Paulo. In Anais do $3^{\circ}$ Simpósio de Redes Hidrológicas, Belo Horizonte, MG.

Qamar, U. M., Azmat, M., Shahid, M. A., Ganora, D., Ahmad, S., Cheema, M. J. M., Faiz, M. A., Sarwar, A., Shafeeque, M., \& Khan, M. I. (2017). Rainfall extremes: a novel modeling approach for regionalization. Water Resourch Management ,31, 1975-1994.

Rocha Neto, B. P., \& Blanco, C. J. C. (2020). Determinação de Curva IDF para o município de Governador Newton Bello no estado do Maranhão. Research, Society and Development, 9(3), 1-12.

Sane, Y., Panthou, G., Bodian, A., Vischel, T., Lebel, T., Dacosta, H., Quantin, G., Wilcox, C., Ndiaye, O., \& Diongue-Niang, A. (2018). Intensity-durationfrequency (IDF) rainfall curves in Senegal. Natural Hazards and Earth System Sciences, 18(7), 1849-1866.

Santos, S. L., Fernandes, V. O., \& Medeiros, Y. D. P. (2019). Sustentabilidade de cidades no contexto da integração entre a gestão dos recursos hídricos e planejamento urbano e territorial. Bahia Análise \& Dados, 29(2), 54-75.

Shrestha, A., Babel, M. S., Weesakul, S., \& Vojinovic, Z. (2017). Developing Intensity-Duration-Frequency (IDF) Curves under Climate Change Uncertainty: The Case of Bangkok, Thailand. Water, 9(2), 1-22.

Silva, S. R., \& Araújo, G. R. S. (2013). Algoritmo para determinação da equação de chuvas intensas. Revista Brasileira de Geografia Física, 6(5), 1371-1383.

Silveira, A. L. L. (2000). Equação para os coeficientes de desagregação de chuva. Revista Brasileira de Recursos Hídricos, 5(4), 143-147.

Sousa, F. R. C., \& Paula, D. P. (2018). Uso da equação de intensidade-duração-frequência de chuvas para bacia do rio Coreaú (Ceará, Brasil). Revista Eletrônica do PRODEMA, 12(1), 96 - 109. 
Research, Society and Development, v. 10, n. 4, e31310414213, 2021

(CC BY 4.0) | ISSN 2525-3409 | DOI: http://dx.doi.org/10.33448/rsd-v10i4.14213

Souza, O. R. M., Scaramussa, P. H. M., Amara, M. A. C. M., Pereira Neto J. A., Pantoja, A. V., \& Sadeck, L. W. R. (2012). Equações de chuvas intensas para o Estado do Pará. Revista Brasileira de Engenharia Agrícola e Ambiental, 16(9), 999-1005.

Svensson, C., Clarke, R. T., \& Jones, D. A. (2007). An experimental comparison of methods for estimating rainfall intensity-duration-frequency relations from fragmentary records. Journal of Hydrology, 341, 79-89.

Teodoro, P. E., Neivock, M. P., Marques, J. R. F., Flores, A. M. F., \& Braga, C. (2014). Influência de diferentes coeficientes de desagregação na determinação de equações IDF para Aquidauana/MS. Revista Eletrrônica de Engenharia Civil, 9(2), 1-9. 